\title{
INTENSIDAD DE PLAGAS Y SUS ENEMIGOS NATURALES EN UNA ASOCIACIÓN DE MAÍZ Y FRÍJOL FERTILIZADOS CON DIFERENTES FORMAS DE LODO DE ALCANTARILLADO
}

\author{
G.L.D. Leite ${ }^{1}$, F.W.S. Silva ${ }^{1}$, C.S. Ferreira ${ }^{1}$, R.E.M. Guañabens ${ }^{1}$, \\ J.A. Lazo $^{2}$, R.A. Sampaio ${ }^{1}$, T.A.R. Nogueira ${ }^{1}$, I.M. Fonseca ${ }^{1}$
}

${ }^{1}$ Universidade Federal de Minas Gerais, Instituto de Ciências Agrárias, Insetário "G.W.G. de Moraes", Av. Universitária, 1000, CEP 39404-006, Montes Claros, MG, Brasil. E-mail: gldleite@ufmg.br

\section{RESUMEN}

Se evaluó la intensidad del ataque de plagas y enemigos naturales en una asociación de maíz (Zea maiz) y fríjol caupí (Vigna unguiculata), así como la incidencia de plagas en granos almacenados de los respectivos cultivos. Los tratamientos consistieron en: Suelo sin fertilización, Encalado y fertilización química, Lodo no higienizado, Lodo higienizado con extracto de neem (Azadirachta indica), Lodo tratado con cal virgen, Compuesto orgánico de lodo y Ipomoea carnea, Compuesto orgánico de lodo y I. carnea + fosfato natural al inicio del compostaje, Lodo no higienizado + fosfato natural en el campo, Lodo higienizado con neem + fosfato natural en el campo, Lodo tratado con cal virgen + fosfato natural en el campo, Compuesto orgánico de lodo y I. carnea + fosfato natural en el campo. Se realizaron observaciones semanales de plagas y enemigos naturales y los datos obtenidos se sometieron a análisis de varianza y la prueba de Scott-Knott a 5\% de probabilidad. El suelo que recibió lodo de alcantarillado tratado con neem fue el que presentó el menor ataque de plagas. Otro tratamiento poco atacado por plagas fue el que recibió lodo de alcantarillado seco higienizado con extracto de neem + aplicación en el campo de fosfato natural reactivo. Por lo tanto, esos dos tratamientos son los recomendados para los productores.

PALABRAS-CLAVE: Insectos, depredadores, Zea mays, Vigna unguiculata, Azadirachta indica.

\section{RESUMO}

INTENSIDADE DE PRAGAS E INIMIGOS NATURAIS EM UM CONSÓRCIO DE MILHO E FEIJÃO ADUBADOS COM DIFERENTES FORMAS DE LODO DE ESGOTO. Avaliou-se a intensidade de pragas e inimigos naturais em um consórcio de milho (Zea maiz) e feijão (Vigna unguiculata), assim como a incidência de pragas em grãos armazenados dos respectivos cultivos. Os tratamentos consistiram em: Solo sem adubação, Calagem e adubação química, Lodo não higienizado, Lodo higienizado com extrato de neem (Azadirachta indica), Lodo tratado com cal virgen, Composto orgânico de lodo e Ipomoea carnea, Composto orgânico de lodo e I. carnea + fosfato natural no início da compostagem, Lodo não higienizado + fosfato natural no campo, Lodo higienizado com neem + fosfato natural no campo, Lodo tratado com cal virgen + fosfato natural no campo, Composto orgânico de lodo e I. carnea + fosfato natural no campo. As avaliações de pragas e inimigos naturais foram semanais e os dados obtidos foram submetidos à análise de variância e ao teste Scott-Knott a 5\% de probabilidade. O tratamento onde se avaliou o lodo higienizado com neem foi o que apresentou o menor ataque de pragas. Outro tratamento pouco atacado por pragas foi o que se avaliou o lodo higienizado com neem e fosfato no solo. Portanto, esses tratamentos são recomendados para os produtores.

PALAVRAS-CHAVE: Insetos, predadores, Zea mays, Vigna unguiculata, Azadirachta indica.

de residuos. El papel de la naturaleza como agente importante del reciclaje llegó a ser insuficiente frente al rápido aumento de la población, lo que

\section{INTRODUCCIÓN}

La actividad humana es generadora permanente generó la necesidad de descontaminación de las aguas y un uso adecuado del producto final de las alcantarillas tratadas (lodo de alcantarillado)

${ }^{2}$ Instituto de Ciencia Animal, San José de las Lajas, La Habana, Cuba. 
(Bettiol; Camargo, 2000; Nascimento et al., 2004) y estos se convirtieron en grandes fuentes de pagos (Bettiol; CAMargo, 2000).

Entre los principales destinos del lodo se encuentran el enterramiento, la incineración, el vertimiento al océano y la aplicación en el suelo, siendo ésta última utilizada para mejorar el reciclaje de nutrientes y materia orgánica, con un efecto favorable sobre la producción de biomasa y de las propiedades químicas y físicas del suelo (PERNIN et al., 2006), promoviendo un mejor desarrollo de las plantas, generando una mayor resistencia al ataque de plagas. SouzA et al. (2004), señalan que la búsqueda de prácticas culturales eficientes pueden asegurar mejoras en la producción, de forma práctica y eficiente en función del uso de insumos, constituyen una opción para el aumento de la producción de alimentos, principalmente en la pequeña propiedad rural.

La asociación del fríjol trepador - caupí (Vigna unguiculata (L.) Walp) e fríjol común (Phaseolus vulgaris L.) con maíz (Zea maiz L.), se realiza en varios países (ARMSTRONG et al., 2008; Geren et al., 2008; DAwo et al., 2009), entre ellos Brasil, principalmente por pequeños productores (CARDOso et al., 2007). El maíz y el fríjol son cultivos donde ya se ha utilizado el lodo de alcantarillado, absorbiéndose bien este biosólido y aumentando la diversidad de enemigos naturales en las plantaciones (Altieri et al., 1990; CIVIDANES; BARBOSA, 2001; GALLO et al., 2002; GONÇALVES; SOUZA-SILVA, 2003).

Enloque conciernea las plagas, Gianolietal.(2006) encontró menor incidencia de Carpophilus sp. (Coleoptera: Nitidulidae) y Pagiocerus frontalis (Fabricius) (Coleoptera: Scolytidae) en maíz asociada con fríjol común, mientras QuiNDERE; SANTOS (1986), señalaron que en la asociación maíz-fríjol caupí, la población de ninfas de Empoasca kraemeri Ross \& Moore (Hemiptera: Cicadellidae), fue menor, en comparación con las ninfas encontradas en monocultivos. Estudios hechos sobre la diversidad de insectos en agroecosistemas demuestran que la asociación de cultivos puede aumentar o disminuir la intensidad de plagas (TEIXEIRA; FrANCO, 2007). En general, se plantea que las poblaciones de insectos fitofagos son frecuentemente más bajas en policultivos que en sistemas de monocultivos (GALLO et al., 2002; GIANOLI et al., 2006), además la asociación puede aumentar a actividad microbiana del suelo e actuar como deterrente contra plantas dañinas e plagas (GEREN et al., 2008).

El estudio de la influencia de abonos orgánicos y fertilidad del suelo en la población de artrópodos es de relevante importancia, ya que teniendo estos índices contabilizados, se pueden usar para la implantación del Manejo Integrado de Plagas - MIP (Altieri; Nicholls, 2003). De forma general, o exceso o deficiencia de algún nutriente puede contribuir al aumento de la intensidad de plagas (GulLAN;
CRANSTON, 2005; Chen et al., 2008a).

La fertilización así como el control de insectosplaga tienen un alto costo monetario y ambiental para la agricultura moderna (AMTMANN et al., 2008). El objetivo de este trabajo fue estudiar la influencia de diferentes formas de lodo de alcantarillado usado como fertilizante orgánico en la intensidad del ataque de plagas y de sus enemigos naturales en una asociación de maíz y fríjol caupí, así como la incidencia de plagas en granos almacenados de los respectivos cultivos.

\section{MATERIALES Y MÉTODOS}

El trabajo se llevó a cabo en lo "Instituto de Ciencias Agrárias da Universidade Federal de Minas Gerais, municipio de Montes Claros ubicado al norte del estado Minas Gerais, Brasil, entre los $4353^{\circ}$ de longitud W y los $16^{\circ}$ latitud S y 43 Altitud: $650 \mathrm{~m}$ ). El norte de Minas Gerais Estado tiene un clima Aw: tropical de sabana, de acuerdo a la clasificación de Köppen, con invierno seco y lluvioso verano, con una precipitación anual de $1.100 \mathrm{~mm}$. La investigación se realizó en un suelo Cambissolo Háplico en una asociación maíz criollo (Zea mays L.) y frijol trepador - caupí (Vigna unguiculata (L.) Walp).

Se empleo un diseño en bloques al azar con seis réplicas y los tratamientos fueron: $\mathrm{T}_{1}$ - Testigo (suelo sin fertilización); $\mathrm{T}_{2}$ - Encalado (3,65 t ha ${ }^{-1}$ de cal dolomítica) y fertilización química convencional $(575 \mathrm{~kg}$ ha $^{-1}$ de sulfato de amonio $+66,7 \mathrm{~kg} \mathrm{ha}^{-1}$ de cloruro de potasio $+500 \mathrm{~kg} \mathrm{ha}^{-1}$ de superfosfato simples); $\mathrm{T}_{3}-$ Aplicación de 16,7 tha-1 delodo de alcantarillado seco no higienizado; $\mathrm{T}_{4}$ - Aplicación de 13,8 t ha ${ }^{-1}$ de lodo de alcantarillado seco higienizado con extracto de neem (Azadirachta indica A. Juss) (Indica: Meliaceae); $\mathrm{T}_{5}$ - Aplicación de 33,2 tha $\mathrm{a}^{-1}$ de lodo de alcantarillado seco tratado con cal virgen; $\mathrm{T}_{6}$ - Aplicación de 28,1 t ha ${ }^{-1}$ compuesto orgánico hecho con lodo de alcantarillado y de Ipomoea carnea subsp. fistulosa Mart.; Choisy (Carnea: Convolvulaceae); $\mathrm{T}_{7}$ - Aplicación de 30,6 t ha ${ }^{-1}$ compuesto orgánico hecho con lodo de alcantarillado y de I. carnea, con adición al inicio del compostaje de $2 \%$ de fosfato natural reactivo; $\mathrm{T}_{8}$ - Aplicación de 14,1 tha ${ }^{-1}$ de lodo de alcantarillado seco no higienizado + Aplicación en el campo de 200 $\mathrm{kg} \mathrm{ha}^{-1}$ de fosfato natural reactivo; $\mathrm{T}_{9}$ - Aplicación de 13,1 t/ ha de lodo de alcantarillado seco higienizado con extracto deneem + Aplicación en el campo de 200 $\mathrm{kg} \mathrm{ha}^{-1}$ de fosfato natural reactivo; $\mathrm{T}_{10}$ - Aplicación de 31,8 t ha $^{-1}$ de lodo de alcantarillado seco tratado con cal virgen + Aplicación en el campo de $200 \mathrm{~kg}$ ha $^{-1}$ de fosfato natural reactivo; $\mathrm{T}_{11}-$ Aplicación de $24,8 \mathrm{t} \mathrm{ha}^{-1}$ compuesto orgánico hecho con lodo de alcantarillado y I. carnea + Aplicación en el campo de $200 \mathrm{~kg} \mathrm{ha}^{-1}$ de fosfato natural reactivo. 
El cultivo se realizó en el período de enero a junio de 2005. Fueron sembradas cinco semillas de maíz y dos de fríjol caupí por surco y 15 días después de la siembra se realizó un raleo dejando una planta de maíz y una de fríjol caupí. Se emplearon parcelas experimentales de $14 \mathrm{~m}^{2}$ y la distancia de siembra fue de $0,5 \mathrm{~m}$ entre plantas y 1,0 $\mathrm{m}$ entre surcos utilizándose 28 plantas de maíz y 28 de fríjol caupí en asociación para las mediciones. Los surcos de los extremos y la primera y última planta de cada surco fueron considerados efecto de bordes, es decir la parcela experimental poseía 4 líneas de maíz y de fríjol caupí, considerándose parcela útil las dos hileras centrales y las cinco plantas de maíz y fríjol en las mismas.

El lodo de alcantarillado deshidratado fue colectado en la Alcantarilla de la Estación - ETE en el municipio de Juramento, MG, la cual es operado por la Copasa, MG, y la macrofita I. carnea adquirida en el depósito delSistema VerdeGrande, donde constituye una importante plaga que aumenta los costos de la conservación del agua que se suministra a la ciudad de Montes Claros. Después de la colecta se tomaron muestras de ambos productos y fueron enviados al laboratorio para determinar, según metodología TEDEsCoetal. (1995), los contenidos de: materia orgánica, $\mathrm{pH}$, humedad, carbono y nitrógeno. El contenido de carbono y nitrógeno se utilizaron para calcular la relación $\mathrm{C} / \mathrm{N}$ en la elaboración del compostaje.

El compostaje de lodo se realizó conjuntamente con la I. carnea la cual fue troceada con un desintegrador eléctrico y tuvo un volumen de $1,5 \mathrm{~m}^{3}$. Se controló diariamente la temperatura y la humedad del material y siempre que fue necesario se realizó una volteo manual de las pilas, utilizándose palas y azadas. La temperatura fue medida a través de barras dehierro introducida en la pila de compostaje en tres puntos específicos: en la superficie, el medioy la base. La humedad fue determinada por el secado en estufa a $105^{\circ} \mathrm{C}$ hasta peso constante. Al final del proceso de degradación, el material fue almacenado por 30 días más para la maduración del compuesto. La macrófita I. carnea mezclada al lodo de alcantarillado presentó buenos resultados en la producción de compuesto orgánico, constituyéndose en una importante fuente de abono para uso agrícola, pudiendo ser explorado por la Copasa, MG.

Las variaciones en las dosis del lodo fueron establecidas conforme su humedad y en la exigencia de nitrógeno para el cultivo del maíz ( $\left.115 \mathrm{~kg} \mathrm{ha}^{-1} \mathrm{de} \mathrm{N}\right)$, y se basó en la disponibilidad de este elemento en el lodo o en el compuesto de lodo y I. carnea. También fue considerada una tasa de mineralización del material del $50 \%$ por año. Los tratamientos higienizados con neem recibieron medio litro de extracto acuoso de hojas por cada litro de lodo de alcantarillado seco. Los tratamientos higienizados con cal virgen fueron tratados en la proporción del 50\% del peso seco de lodo + un 50\% del peso seco de cal virgen, mezclados a un $70 \%$ de humedad del lodo. En el tratamiento con encalaje y fertilización química fueron aplicados 3,65 t/ ha de cal dolomítica, para elevación de la saturación de bases para un $70 \%$, y $575,66,7$ y $500 \mathrm{~kg} \mathrm{ha}^{-1}$ de sulfato de amonio, cloruro de potasio y superfosfato simples, respectivamente, con base en el análisis del sueloy en la recomendación de fertilización para el cultivo del maíz.

Los contenidos totales de los nutrientes en las diferentes formas delodo dealcantarilladoutilizadas en el experimento fueron determinados en el extracto ácido (ácido nítrico y ácido perclórico) (TEDEsco et al., 1995). En el suelo, fueron determinados en muestras colectadas a la profundidad de 0 la $20 \mathrm{~cm}$ : el $\mathrm{pH}$ y los contenidos de $\mathrm{P}, \mathrm{K}, \mathrm{Ca}, \mathrm{Mg}, \mathrm{Al}, \mathrm{H}+\mathrm{Al}$ y materia orgánica, de acuerdo con las metodologías descritas por TEDESCO et al. (1995). Las cosechas de fríjol y fueron realizadas, respectivamente, a los 90 y 160 días después de la plantación, considerándose para la misma un 13\% de humedad en los granos.

Las evaluaciones entomológicas de las plagas (insectos y ácaros) y enemigos naturales (insectos depredadores y arañas) fueron semanales por medio de conteo directo (SiLva et al., 2009), donde se anotó en el maíz el porcentaje de defoliación y la presencia de artrópodos en la primera hoja expandida de cada planta y espigas, siendo evaluadas 10 plantas/ parte. En el fríjol las evaluaciones se realizaron durante todo el ciclo de la planta, sin embargo, en la fase inicial de estas, se evaluó el porcentaje de daño por crisomélidos (Coleoptera) y la población de artrópodos (plagas y enemigos naturales) en una hoja/planta, mientras que en las diferentes etapas del desarrollo fenológico, este indicador se evaluó en una hoja de cada parte de la planta (apical, media y basal) y el porcentaje de daño por artrópodos en las vainas, en 10 plantas/parcela (Silva et al., 2009). En estas fases también se evaluó por medio de lupa binocular $(40$ x) la cantidad de ácaros presentes en la parte basal de tres hojas/planta en tres plantas/ parcela utilizando de igual forma cada parte de la planta (apical, media y basal).

Los granos fueron cosechados 90 y 160 días después de la plantación para el fríjol caupí y maíz, respectivamente, y fueron llevados al laboratorio de entomologia, donde se realizó la separación de 50 granos para cada una de las especies según los tratamientos, las cuales se colocaron, independientemente, en pomos plástico de color blanco $(11,5$ x 8,5 $\mathrm{cm})$ cerrados herméticamente con tapa plástica, para la evaluación de granos almacenados, por un periodo de 20 semanas, la cual se desarrollo de forma semanal (Almeida et al., 2005). Los datos obtenidos, tanto en campo como en laboratorio fueron transformados en $\sqrt{ } x+0,5 x$ o arcoseno $x$ (para los datos en porcentaje), $y$ 
luego la prueba de la normalidad y la homogeneidad de las varianzas, al analisis de varianza y a la prueba de Scott-Knott a un 5\% de probabilidad.

\section{RESULTADOS Y DISCUSIÓN}

No se detectó efecto significativo entre los diferentes tratamientos para la producción de maíz y fríjol caupí (Tabla 1), debido posiblemente a que el área del experimento fue utilizada con anterioridad por otros cultivos y esto pudo provocar un efecto residual de nutrientes, según lo observado por los elevados valores de los elementos químicos en el suelo (Tabla 2).

El menor número de larvas de Spodoptera frugiperdaSmith (Lepidoptera: Noctuidae) y de sus daños en las hojas del maíz (Tabla 1) pueden ser atribuidas, probablemente, a los contenidos más elevados de calcio (Ca) en el suelo (Tabla 2) y en el lodo de alcantarillado tratado con cal virgen (Tabla 2); y de potasio (K) en los tratamientos cuyo suelos fueron tratados con encalaje + fertilización convencional, con lodo de alcantarillado seco higienizado con extracto de neem + fosfato natural reactivo aplicado en el suelo y con compuesto orgánico de lodo de alcantarillado y I. carnea + fosfato natural (Tabla 2).

El mayor ataque de $S$. frugiperda en el maíz fue observado en el tratamiento donde se aplicó 14,1 t/ ha de lodo de alcantarillado seco no higienizado + aplicación en campo de $200 \mathrm{~kg} /$ ha de fosfato natural reactivo (Tabla 1) y puede estar relacionado con los menores contenidos de $\mathrm{K}$ y $\mathrm{Ca}$ en el suelo (Tabla 2) y en el lodo de alcantarillado no higienizado + fosfato natural en el suelo (Tabla 2). El potasio (K) está asociado a la resistencia de la plantas a insectos, principalmente los chupadores (SILva et al., 2009), ya que este elemento forma parte en la síntesis de la RNA polimerasa enzima responsable por la ruta de los aminoácidos libres en la savia necesarios en la formación de la proteína (TAIZ; ZEIGER, 2004; WALTER; Difonzo, 2007). El Calcio (Ca) también es un elemento nutricional importanteen la resistencia delas plantas al ataque de plagas (SiLva et al., 2009), pues él es parte constituyente de la lignina y la lámina media de la pared celular de los vegetales, confiriendo mayor dureza (TAIZ; ZeIGER, 2004).

Los menores ataques de Helicoverpa zea (Bod.) (Lepidoptera: Noctuidae) encontrados en las espigas de maíz en los tratamientos donde se utilizaron lodo dealcantarillado tratado con neem y lodo de alcantarillado tratado con cal virgen + fosfato natural en el suelo (Tabla 1) son debidos, probablemente, al bajo contenido de fósforo (P) y de K en el suelo (Tabla 2), lo que pueden provocar espigas menos atractivas para esta plaga, pues estos elementos químicos son importantes en la ruta metabólica de las proteínas (MiYASAKA et al., 2007; HoPMANs et al., 2008). Además de eso, el tratamiento que recibió lodo de alcantaril- lado tratado con neem presentó menor contenido de nitrógeno $(\mathrm{N})$ en el lodo de alcantarillado (Tabla 2). Chen et al. (2008b) relató que Spodoptera exigua (Hübner) (Lepidoptera: Noctuidae) alimentada en plantas con menor calidad nutricional, o sea, baja cantidad de $\mathrm{N}$ es afectada en su desarrollo. La menor incidencia de $H$. zea encontrada en las espigas de maíz en los tratamientos donde se realizó encalaje + fertilización convencional, a pesar de los altos contenidos de $\mathrm{P}$ y de $\mathrm{K}$ en el suelo, la relacionamos quizás con el hecho de que la fertilización química pudo no haber. propiciado un adecuado balance nutricional comparado con los que utilizaron lodo de alcantarillado (CHABOussou, 1987). El mayor ataque de $H$. zea observado en la testigo puede deberse, probablemente, al estrés nutricional de la planta, provocando que sea más susceptible (CHABOUssou, 1987).

La mayor desfoliación de fríjol en el testigo por Diabrotica speciosa Germar y Cerotoma arcuatus Olivier (Coleoptera: Chrysomelidae) (Tabla 1) se debe probablemente al estrés de la planta, lo que resultó en mayor susceptibilidad de la misma o tal vez por la menor área foliar, provocando un efecto de concentración en el daño de desfoliación (LEITE et al., 2003). Ya en el suelo que fue tratado con lodo alcantarillado no higienizado + fosfato natural en el suelo, la mayor desfoliación posiblemente se deba al mayor contenido de $P$ en el suelo y a los contenidos medios de N, P y K en el lodo (Tablas 1 y 2), lo que puede influir en un mayor contenido de proteína en la hojas.

En el tratamiento que se aplicó lodo de alcantarillado tratado con cal virgen + fosfato natural en el suelo, a pesar de bajos contenidos de $\mathrm{K}$ y $\mathrm{P}$, tanto en el suelo como en el lodo, presentó los mayores contenidos de $\mathrm{N}$ en el lodo, lo que posibilito quizás disponer de hojas más nutritivas, atrayendo los crisomélidos. Es posible que la fertilización deficiente en $\mathrm{N}$ afecte el balance de la relación proteína: carbohidrato o reduzca compuestos defensivos de la planta (CHEN et al., 2008b), tornándola más susceptible al ataque de plagas. La menor desfoliación ocasionada por estos insectos se observa en el tratamiento que recibió encalaje + fertilización convencional, a pesar de los altos contenidos de $\mathrm{P}$ y de K en el suelo, probablemente debido al desfavorable balance químico de la planta para los insectos masticadores con la fertilización química comparada con el testigo (estrés nutricional) y a los demasiados tratamientos con lodo de alcantarillado y compuesto orgánico. La menor desfoliación en los tratamientos con lodo de alcantarillado no higienizado, con lodo de alcantarillado tratado con neem y con lodo de alcantarillado seco higienizado con extracto de neem + fosfato natural reactivo aplicado en el suelo, presentaron menores contenidos de $\mathrm{P}$ (en el suelo) y 


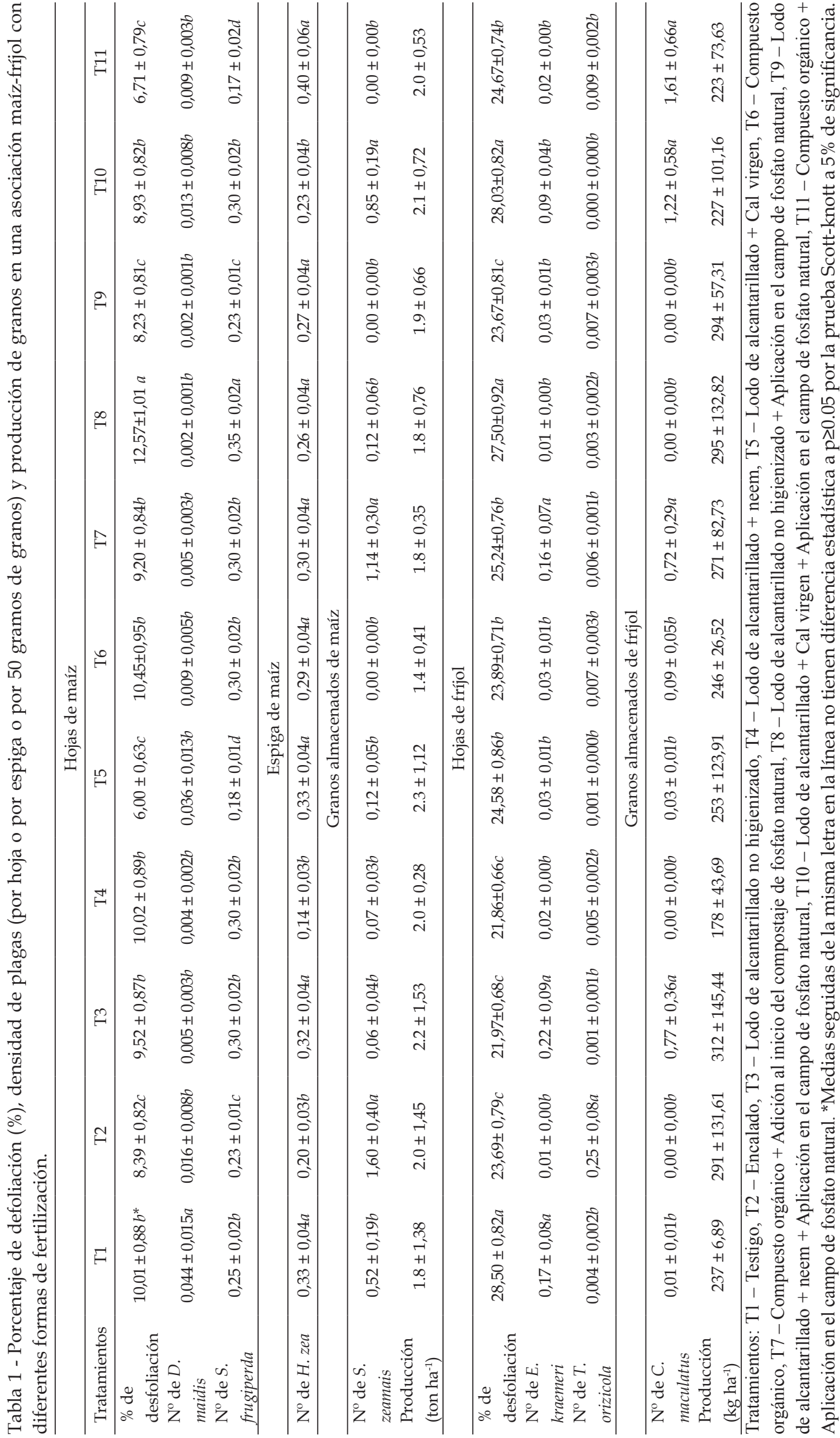


Tabla 2 - Caracterización química y física del suelo en el área experimental, efecto de los tratamientos en la composición química del suelo, y composición química y pH en los materiales orgánicos utilizados en los tratamientos.

\begin{tabular}{|c|c|c|c|}
\hline \multicolumn{4}{|c|}{ Caracterización química y física del suelo en el área experimental* } \\
\hline Características del suelo & Valor & Características del suelo & Valor \\
\hline pH en agua & 5,4 & $\mathrm{t}\left(\mathrm{cmol}_{\mathrm{c}} \mathrm{dm}^{-3}\right)$ & 6,00 \\
\hline P-Mehlich 1 (mg dm $\left.{ }^{-3}\right)$ & 4,2 & $\mathrm{~m}(\%)$ & 3 \\
\hline $\mathrm{K}\left(\mathrm{mg} \mathrm{dm}^{-3}\right)$ & 76 & $\mathrm{~T}\left(\mathrm{cmol}_{\mathrm{c}} \mathrm{dm}^{-3}\right)$ & 14,59 \\
\hline $\mathrm{Ca}\left(\mathrm{cmol}_{\mathrm{c}} \mathrm{dm}^{-3}\right)$ & 4,10 & $\mathrm{~V}(\%)$ & 40 \\
\hline $\mathrm{Mg}\left(\mathrm{cmol}_{\mathrm{c}} \mathrm{dm}^{-3}\right)$ & 1,50 & Mat. Orgánica (dag kg-1) & 5,58 \\
\hline $\mathrm{Al}\left(\mathrm{cmol}_{\mathrm{c}} \mathrm{dm}^{-3}\right)$ & 0,20 & Arena $($ dag kg-1) & 40 \\
\hline $\mathrm{H}+\mathrm{Al}\left(\mathrm{cmol}_{\mathrm{c}} \mathrm{dm}^{-3}\right)$ & 8,80 & Limo $($ dag kg-1) & 30 \\
\hline Suma de Bases $\left(\mathrm{cmol}_{\mathrm{c}} \mathrm{dm}^{-3}\right)$ & 5,80 & Arcilla (dag kg-1) & 30 \\
\hline
\end{tabular}

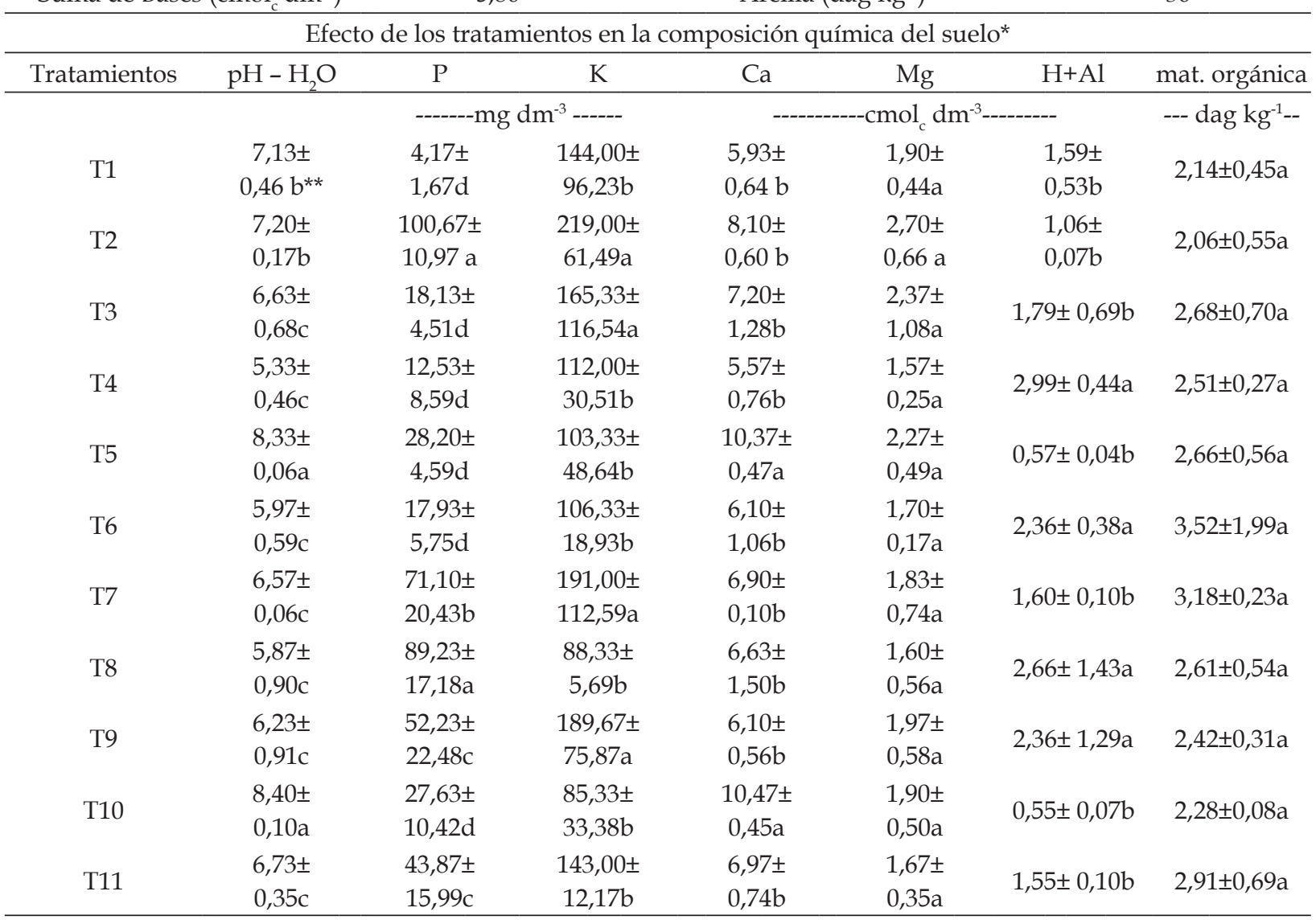

\begin{tabular}{|c|c|c|c|c|c|c|c|c|c|}
\hline \multicolumn{10}{|c|}{ Composición química y pH en los materiales orgánicos utilizados en los tratamientos } \\
\hline Tratamientos & $\mathrm{pH}-\mathrm{H}_{2} \mathrm{O}$ & $\mathrm{N}$ & $\mathrm{P}$ & K & $\mathrm{Ca}$ & $\mathrm{Mg}$ & $S$ & C.O. & $\mathrm{C} / \mathrm{N}$ \\
\hline \multicolumn{10}{|c|}{----- $\mathrm{mg} \mathrm{kg}^{-1}$} \\
\hline T3 & 4,40 & 10,4 & 2,9 & 5,8 & 7,0 & 3,2 & 11,5 & 6,55 & 6,29 \\
\hline $\mathrm{T} 4$ & 4,75 & 7,8 & 3,0 & 5,6 & 6,5 & 3,0 & 9,2 & 7,02 & 9,00 \\
\hline $\mathrm{T} 5$ & 12,02 & 14,0 & 1,7 & 4,0 & 146,6 & 2,6 & 6,3 & 3,74 & 2,67 \\
\hline T6 & 5,06 & 10,6 & 1,3 & 4,4 & 14,6 & 1,6 & 6,5 & 12,6 & 11,88 \\
\hline $\mathrm{T} 7$ & 4,56 & 10,9 & 3,7 & 5,8 & 18,5 & 2,2 & 6,1 & 12,00 & 11,00 \\
\hline
\end{tabular}

Tratamientos: T1 - Testigo, T2 - Encalado, T3 - Lodo de alcantarillado no higienizado, T4 - Lodo de alcantarillado + neem, T5 - Lodo de alcantarillado + Cal virgen, T6 - Compuesto orgánico, T7 - Compuesto orgánico + Adición al inicio del compostaje de fosfato natural, T8 - Lodo de alcantarillado no higienizado + Aplicación en el campo de fosfato natural, T9 - Lodo de alcantarillado + neem + Aplicación en el campo de fosfato natural, T10 - Lodo de alcantarillado + Cal virgen + Aplicación en el campo de fosfato natural, T11 - Compuesto orgánico + Aplicación en el campo de fosfato natural.

*Metodología de la Embrapa (1997).

** Medias con letras diferentes dentro de una misma columna difieren a $\mathrm{p}<0.05$ por la prueba de Scott-Knott a $5 \%$ de significancia. 
de $\mathrm{N}$ (en el lodo), a pesar del elevado contenido de $\mathrm{K}$, resultando, posiblemente, en un alimento menos nutritivo en cuanto a proteína foliar.

Elinsectoque más seencontró en almacenamiento de los granos de maíz fue Sitophilus zeamais Motsch. (Coleoptera: Curculionidae), en los tratamientos que recibieron encalaje + fertilización convencional y compostaje de lodo de alcantarillado y de I. carnea + fosfato natural en el compostaje (Tabla 1), debido quizás, a los altos contenidos de $\mathrm{P}$ y de K en el suelo $\mathrm{y}$, probablemente, más proteína fijada en los granos y debido también al bajo contenido de Ca en el suelo, que puede provocar granos menos duros (Tabla 2). Hecho similar fue observado para el mayor ataque de Callosobruchus maculatus Fabricius (Coleoptera: Bruchidae) en granos de fríjol en el almacenamiento de los tratamientos que recibieron lodo de alcantarillado no higienizado, compostaje delodo de alcantarillado y I. carnea + fosfato natural en el compuesto y compostaje de lodo alcantarillado y $I$. carnea + fosfato natural en el suelo (Tabla 1), en lo cual fue detectado menor contenido de $\mathrm{Ca}$ en el suelo y, conjuntamente con esto, una concentración intermediaria de $\mathrm{N}$ en el lodo y en el compostaje de estos tratamientos (más proteína en el grano) (Tabla 2). Ya en el caso del tratamiento que se utilizó lodo de alcantarillado tratado con cal virgen + fosfato natural en el suelo, los mayores ataques de $S$. zeamais y de $C$. maculatus (Tabla1) ocurrieron, probablemente, al alto contenido de $\mathrm{N}$ en el compuesto, lo que debe provocar granos de fríjol con mayor contenido proteico, a pesar del mayor contenido de Ca (granos más duros), de los bajos contenidos de $\mathrm{P}$ en el suelo y en el compostaje y contenidos mediano y bajo de K en el suelo y en el compostaje, respectivamente (Tabla 2).

Los mayores ataques de Dalbulus maidis DeLong; Wolcott(DeLong; Wolcott) (Hemiptera:Cicadellidae) encontrados en las hojas de maíz y de Empoasca kraemeri Ross; Moore (Hemiptera: Cicadellidae) en las hojas de fríjol en el testigo (Tabla 1) se deben, probablemente, a los bajos contenidos de $\mathrm{P}$ y de $\mathrm{K}$ en el suelo comparado a los demás tratamientos y al nitrógeno residual relativamente alto en el suelo, que puede causar una mayor concentración de aminoácidos libres en la savia, y bajo contenido de Ca en el suelo, responsable por la resistencia de la pared celular (dificulta la alimentación de insectos chupadores) (Tabla 2). MIYASAKA et al. (2007) relatan que el desbalance entre $\mathrm{N}$ e $\mathrm{K}$ (menores cantidades de $\mathrm{K}$ y mayores de $\mathrm{N}$ ) aumento el daño provocado por Sipha flava (Forbes) (Hemiptera: Aphididae), consecuencia de la baja síntesis proteica y mayor concentración de aminoácidos libres en la savia de la planta. Hopmans et al. (2008) encontraron que la aplicación de $\mathrm{N}$ aumento la herbivoria por Essigella californica (Essig.) (Hemiptera: Aphididae) en condición de deficiencia de P. Estos autores sugieren queel elevado ataque del pulgón fue consecuencia de una mayor palatabilidad de la planta en condiciones de desbalance nutricional, limitado por suplemento de P. Además de esto, puede se han producido un efecto de concentración de insectos en las hojas de fríjol y de maíz debido a que las plantas presentaron un menor crecimiento comparado con los otros tratamientos (LeiTe et al., 2003). Este mismo análisis puede ser utilizado en el tratamiento que recibió lodo de alcantarillado tratado con cal virgen, pues el mayor ataque de $D$. maidis puede asociarse quizás al alto contenido de $\mathrm{N}$ y los bajos contenidos de $\mathrm{P}$ en el lodo y de $\mathrm{P}$ y K en el suelo.

Elmayor número de E. kraemerienlos tratamientos que recibieron lodo de alcantarillado no higienizado y compostaje de lodo de alcantarillado y de I. carnea + fosfato natural añadido al compostaje (Tabla 1) se deben, probablemente, al elevado contenido de $\mathrm{K} y$ bajos contenidos de $\mathrm{P}$ y de $\mathrm{Ca}$ en el suelo y valores intermediarios de $\mathrm{N}$ en el lodo y en el compostaje y mediano a bajo de Ca (Tabla 2). Este balance nutricional se debe reflejar, quizás en una mayor concentración de aminoácidos libres en la savia de las plantas y menor resistencia de pared celular, favoreciendo el ataque de E. kraemeri.

Seobservó un mayor número de Tagosodes orizicola Muir (Hemiptera: Delphacidae) en las hojas de fríjol en el suelo donde se aplicó el encalaje + fertilización química (Tabla 1) debido, probablemente, al bajo contenido de $\mathrm{Ca}$ en el suelo (pared celular menos dura) $\mathrm{y}$, a pesar de los altos contenidos de $\mathrm{P}$ y de $\mathrm{K}$ (Tabla 2), lo que reduciría la concentración de aminoácidos libres en la savia, fue fertilizado con formulación química, lo que hace disponible rápidamente los nutrientes para la planta, desequilibrándola y haciéndola más susceptible al ataque de este insecto chupador (Chaboussou, 1987).

También fueron observados otros insectos fitófagos y ácaros en las hojas de maíz [E. kraemeri, Rhopalosiphum maidis Fitch (Homoptera: Aphididae), Phthia picta Drury (Hemiptera: Coreidae)] y en las hojas de fríjol [T. orizicola, Euxesta sp. (Diptera: Otitidae), Lagria villosa Fabr. (Coleoptera: Lagriidae), Bemisia argentifolii Bellows; Perring (Hemiptera: Aleyrodidae), Rhopalosiphum maidis Fitch (Hemiptera: Aphididae), Liriomyza sp. (Diptera: Agromyzidae), Frankliniella schulzei Trybom (Thysanoptera: Thripidae), Tetranychus ludeni Zacher y T. urticae Koch (Acari: Tetranychidae), Polyphagotarsonemus latus Banks (Acari: Tarsonemidae)], pero en todos los casos estuvieron en una media de cuatro insectos en 1000 hojas de maíz y uno en cada 100 hojas de fríjol y, debido a esto, probablemente, no se detectó efecto de los tratamientos sobre ellos. En las vainas de fríjol fueron encontrados daños por crisomélidos $(23.87 \pm 0.0098)$ y por los insectos: Aetalion reticulatum L. (Homoptera: Aetalionidae) $(0.0012 \pm 0.0008) ; D$. 
speciosa $(0.006 \pm 0.002)$; C. arcuatus $(0.0006 \pm 0.0006)$ y $L$. villosa $(0.0018 \pm 0.0010)$, pero tampoco se detectó efecto de los tratamientos.

Los enemigos naturales, observados en maíz, cinco en cada 1.000 hojas, fueron [Syrphus sp. (Diptera: Syrphidae), Orius sp. (Heteroptera: Anthocoridae), Zelus sp.(Heteroptera: Reduviidae), Vespidae(Hymenoptera), Cicloneda sanguinea L. (Coleoptera: Coccinellidae), Chrysoperlasp.(Neuroptera: Chrysopidae), Doru luteipes Scudder (Dermaptera: Forficulidae) y arañas] y en fríjol, ocho en cada 10.000 hojas fueron [D. luteipes, Vespidae, C. sanguinea, Zelus sp. y arañas]. En ambos casos no fueron afectados por los tratamientos ni se correlacionaron con las plagas debido a sus bajas densidades.

En breve, el suelo que recibió lodo de alcantarillado tratado con neem fue el que presentó el menor ataque de plagas. Otro tratamiento poco atacado por plagas fue el que recibió lodo de alcantarillado seco higienizado con extracto de neem + aplicación en el campo de fosfato natural reactivo. Por lo tanto, esos dos tratamientos son los recomendados para los productores.

\section{REFERENCIAS}

ALMEIDA, C.I.M.; AMORIN, C.A.D.; LEITE, G.L.D.; ROCHA, S.L.; FERNANDES, L.A. Efeito de adubações fosfatadas e da ausência de rotações sobre pragas do milho e seus inimigos naturais e efeito residual de um piretróide sobre pragas de grãos de milho em armazém. Agronomia Lusitana, v.51, p.59-68, 2005.

ALTIERI, M.A.; NICHOLLS, C.I. Soil fertility management and insect pests: harmonizing soil and plant health in agroecosystems. Soil Tillage Research, v.72, p.203-211, 2003.

ALTIERI, M.A.; GLASER, D.L.; SCHMIDT, L.L. Diversification of agroecosystems for insect pest regulation: experiments with collards. In: GLIESSMAN, S.R. (Ed.). Agroecology, researching the ecological basis for sustainable agriculture. New York: Springer-Verlag, 1990. p.70-82.

AMTMANN, A.; TROUFFLARD, S.; ARMENGAUD, $P$. The effect of potassium nutrition on pest and disease resistance in plants. Physiologia Plantarum, v.133, p.682691, 2008.

ARMSTRONG, K.L.; ALBRECHT, K.A.; LAUER, J.G.; RIDAY, H. Intercropping corn with lablab Bean, velvet Bean, and scarlet runner bean for forage. Crop Science, v.48, p.371-379, 2008.

BETTIOL, W.; CAMARGO, O.A. Impacto ambiental do uso agrícola do lodo de esgoto. Jaguariúna: EMBRAPA Meio Ambiente, 2000. 312p.

CARDOSO, E.J.B.N.; NOGUEIRA, M.A.; FERRAZ,
S.M.G. Biological $\mathrm{N}_{2}$ fixation and mineral $\mathrm{n}$ in common bean-maize intercropping or sole cropping in southeastern Brazil. Experimental Agriculture, v.43, p.319-330, 2007. CHABOUSSOU, F. Plantas doentes pelo uso de agrotóxicos: a teoria da trofobiose. Porto Alegre: Ed. L\&M, 1987. $253 p$.

CHEN, Y.; RUBERSON, J.R.; OLSON, D.M. Nitrogen fertilization rate affects feeding, larval performance, and oviposition preference of the beet armyworm, Spodoptera exigua, on cotton. Entomologia Experimentalis et Applicata, v.126, p.244-255, 2008b.

CHEN, Y.; SCHMELZ, E.A.; WÄCKERS, F.; RUBERSON, J.R. Cotton plant, Gossypium hirsutum L., defense in response to nitrogen fertilization. Journal of Chemical Ecology, v.34, p.1553-1564, 2008a.

CIVIDANES, F.J.; BARBOSA J.C. Efeitos do plantio direto e da consorciação soja-milho sobre inimigos naturais e pragas. Pesquisa Agropecuária Brasileira, v.36, p.235-241, 2001.

CORREAA, J.B.; REIS JÚNIOR, R.A.; CARVALHO, J.G.; GUIMARÃES, P.T.G. Avaliação da fertilidade do solo e do estado nutricional de cafeeiros do sul de Minas Gerais. Ciência Agrotécnica, v.25, p.1279-1286, 2001.

DAWO, I.M.; WILKINSON, J.M.; PILBEAM, D.J. Interactions between plants in intercropped maize and common bean. Journal of the Science of Food and Agriculture, v.89, p.41-48, 2009.

GALLO, D.; NAKANO, O.; SILVEIRA NETO, S.; CARVALHO, R.P.L.; BATISTA, G.C.; BERTI FILHO, E.; PARRA, J.R.P.; ZUCCHI, R.A.; ALVES, S.B.; VENDRAMIM, J.D.; MARCHINI, L.C.; LOPES, J.R.S.; OMOTO, C. Entomologia agrícola. Piracicaba: FEALQ, 2002. 920p.

GEREN, H.; AVCIOGLU, R.; SOYA, H.; KIR, B. Intercropping of corn with cowpea and bean: biomass yield and silage quality. African Journal of Biotechnology, v.7, p.4100-4104, 2008.

GIANOLI, E.; RAMOS, I.; ALFARO-TAPIA, A.; VALDÉZ, Y.; ECHEGARAY, E.R.; YÁBAR, E. Benefits of a maize-beanweeds mixed cropping system in Urubamba Valley, Peruvian Andes. International Journal of Pest Management, v.52, p.283-289, 2006.

GONÇALVES, P.A.S.; SOUSA-SILVA, C.R. Efeito de espécies vegetais em bordadura em cebola sobre a densidade populacional de tripes e sirfídeos predadores. Horticultura Brasileira, v.21, p.731-733, 2003.

GULLAN, P.J.; CRANSTON, P.S. The insects: an outline of entomology. Oxford: Blackwell Publishing, 2005. $505 p$.

HOPMANS, P.; COLLETT, N.C.; SMITH, I.W.; ELMS, S.R. Growth and nutrition of Pinus radiata in response 
to fertilizer applied after thinning and interaction with defoliation associated with Essigella californica. Forest Ecology and Management, v.255, p.2118-2128, 2008. LEITE, G.L.D.; COSTA, C.A.; ALMEIDA, C.I.M.; PICANÇO, M. Efeito da adubação sobre a incidência de traça-do-tomateiro e alternaria em plantas de tomate. Horticultura Brasileira, v.21, p.448-451, 2003.

LIMA, R.R.; COSTA, J.P.C. da Coleta de plantas de cultura pré-colombiana na Amazônia brasileira: I. Metodologia e expedições realizadas para coleta de germoplasma. Belém: EMBRAPA-CPATU, 1997. 148p. (EMBRAPA-CPATU, Documentos, 99).

MIYASAKA, S.C.; HANSEN, J.D.; MCDONALD, T.G.; FUKUMOTO, G.K. Effects of nitrogen and potassium in kikuyu grass on feeding by yellow sugarcane aphid. Crop Protection, v.26, p.511-517, 2007.

NASCIMENTO, C.W.A.; BARROS, D.A.S.; MELO, E.E.C.; OLIVEIRA, A.B. Alterações químicas em solos e crescimento de milho e feijoeiro após aplicação de lodo de esgoto. Revista Brasileira de Ciência do Solo, v.28, p.385-392, 2004.

PERNIN, C.; CORTET, C.; JOFFRE, R.; PETIT, J.L.; TORRE, F. Sewage sludge effects on mesofauna and cork oak (Quercus suber L.) leaves decomposition in a mediterranean forest firebreak. Journal of Environmental Quality, v.35, p.2283-2292, 2006.

QUINDERE, M.A.W.; SANTOS, J.H.R. Efeito de época relativa de plantio no consórcio milho $x$ caupi sobre a presença de insetos úteis e o manejo econômico das pragas. Pesquisa Agropecuária Brasileira, v.21, p.355-368, 1986.
SILVA, F.W.S.; LEITE, G.L.D.; GUANABENS, R.E.M.; MARTINS, E.R.; MATIOLI, A.L.; FERNANDES, L.A. Nutrientes afetando as mudas de alecrim-pimenta (Lippia sidoides Cham.) e seus artrópodes. Revista Brasileira de Plantas Medicinais, v.11, p.18-23, 2009.

SOUZA, M.L.O.; TÁVORA, F.J.A.F.; BLEICHER, E.; PITOMBEIRA J.B. Efeito do consórcio do milho (Zea mays L.) com o feijão-de-corda (Vigna unguiculata (L.) Walp.) no rendimento de grãos, uso eficiente da terra e ocorrência de pragas. Revista Ciência Agronômica, v.35, p.196-205, 2004.

TAIZ, L.; ZEIGER, E. Fisiologia vegetal. Porto Alegre: Artmed, 2004. 719p.

TEDESCO, M.J.; GIANELLO, C.; BISSANI, C.A.; BOHNEN, H.; VOLKWEISS, S.J. Análise de solo, plantas e outros materiais. 2.ed. Porto Alegre: Departamento de Solos/UFRGS, 1995. 174p. (Boletim Técnico, 5).

TEIXEIRA, M.L.F.; FRANCO, A.A. Infestação por larvas de Cerotoma arcuata (Olivier) (Coleoptera: Chrysomelidae) em nódulos de feijoeiro em cultivo com cobertura morta ou em consórcio com milho ou com caupi. Ciência Rural, v.37, p.1529-1535, 2007.

WALTER, A.J.; DIFONZO, C.D. Soil potassium deficiency affects soybean phloem nitrogen and soybean aphid populations. Environmental Entomology, v.36, p.26-33, 2007.

Recibido 11/8/10

Aceptado 12/5/11 
